\title{
Anesthesia for Cardiac Surgery during COVID-19 Pandemic
}

\author{
Salome Jeyabalan ${ }^{1} \quad$ Kedar Bangal $^{1} \quad$ Anuja Kulkarni ${ }^{1} \quad$ Maithriye Kavishree ${ }^{1}$ Sujani Kola ${ }^{1}$ \\ Mehak Mahajan ${ }^{1} \quad$ Friday Okonna ${ }^{1}$ Hema C. Nair ${ }^{1}$ Keshava Murthy $S^{1} \quad$ Muralidhar Kanchi ${ }^{1}$
}

${ }^{1}$ Narayana Institute of Cardiac Sciences, Narayana Health City, Bangalore, Karnataka, India

\begin{abstract}
Address for correspondence Salome Jeyabalan, DA, MD, DM, Narayana Institute of Cardiac Sciences, Narayana Health City, \#258/A, Bommasandra Industrial Area, Anekal Taluk, Bangalore 560 099, Karnataka, India (e-mail: salome.jeyabalan@gmail.com).
\end{abstract}

\begin{abstract}
Keywords

- COVID-19

- personal protective equipment (PPE)

- aerosol-generating procedure (AGP)

- transesophageal echocardiography (TEE)

The new coronavirus disease 2019 (COVID-19) is an infectious disease caused by the severe acute respiratory syndrome coronavirus 2 (SARS-CoV-2). Patients with COVID-19 can progress from asymptomatic or mild illness to hypoxemic respiratory failure to multisystem organ dysfunction and death. Healthcare workers, particularly anesthesiologists, are at increased risk since their airway management expertise is required in situations where suspected or confirmed cases of COVID-19 require surgical procedures and in critical care settings. Such patients undergoing surgery have a higher perioperative morbidity and mortality. Additionally, aerosol-generating procedures place the operating room staff at high risk of contracting the COVID-19 infection. Here, we present a review of COVID-19 management, particularly in the perioperative setting. In addition, this article highlights specific concerns with the use of transesophageal echocardiography and the precautions to be taken during cardiopulmonary resuscitation. This review article is based on this institutional protocol supported by literature from recent publications and guidelines from major health organizations on COVID-19.
\end{abstract}

\section{Introduction}

A new type of severe acute respiratory syndrome began in Wuhan, Hubei Province, China in late 2019. This outbreak caught international attention in December 2019 was quickly attributed to a novel coronavirus, and it was named "Corona Virus Disease 2019" (COVID-19) by the World Health Organization on February 12, 2020. This viral disease has spread worldwide affecting all continents except Antarctica and continues to broaden geographically contributing to the increasing number of infected cases and death. As of this writing, the number of people infected worldwide has surpassed 1,993,529 with more than 210 countries affected and 227,638 patients have died as of April 29, 2020. ${ }^{1}$

\section{Transmission and Clinical Course}

The virus first possibly acquired animal-to-human transmission from the wet market of Wuhan, China, in December 2019 and it eventually sustained human-to-human transmission via droplet infection, direct contact, or from fomites. ${ }^{2}$

The incubation period of this viral illness is around 2 to 14 days and the period from the onset of symptoms to death ranges from 6 to 41 days with a median of 14 days. This period is dependent on the patients' age and the status of the immune system (shorter among patients older than 70 years). Risk factors include male gender and patients with comorbidities such as cardiovascular disease, diabetes, chronic pulmonary disease, smoking, and hypertension. ${ }^{3}$ Symptoms include fatigue, fever, headache, dry cough, myalgia, dyspnea, and other symptoms such as diarrhea, nausea, and new loss of taste and smell. The infected patients can have unexpected progression to acute respiratory distress syndrome, renal failure, cardiac injury, and death. Blood investigations reveal lymphopenia, elevated lactate dehydrogenase, high-sensitive cardiac troponin, and prolonged prothrombin time. Imaging findings include consolidation in chest X-ray and ground glass opacities on computed tomography of thorax. ${ }^{3-5}$
DOI https://doi.org/

10.1055/s-0040-1713538 ISSN 2457-0206.
License terms

() (1) $\ominus \circledast$ 


\section{Prevention and Treatment}

Hand hygiene, social distancing, and isolation remain the mainstay of containing COVID-19. Other than supportive treatment with oxygen and mechanical ventilation, antiviral medications are being tested for their effectiveness against COVID-19. Current experimental treatment options include remdesivir, ribavirin, interferon- $1 \beta$, chloroquine, and convalescent plasma. Effective and definitive medical regimens to combat this viral illness have not been developed yet and vaccine development is underway. ${ }^{4}$

\section{Infection Control}

In addition to standard precautions, specific measures to contain droplet spread should be applied to patients who are suspected or confirmed to have COVID-19. Aerosolgenerating procedures (AGP) induce tiny droplets of approximately 5 micron in size that can travel farther and stay suspended in air longer compared to larger droplets. High AGP include mask ventilation, endotracheal intubation, fiberoptic or rigid bronchoscopy, noninvasive ventilation, high flow nasal oxygen delivery, oral and tracheal suctioning, nebulization, cardiopulmonary resuscitation (CPR), and extubation. ${ }^{5}$ Hence, caring for suspected and confirmed cases requires concerted efforts from all healthcare workers (HCWs) and frontline hospital staff across all disciplines. Recommended measures include staff education and training, developing institutional workflow guidelines for safe handling of suspected cases, facilitating triage for prompt diagnosis, training all the concerned hospital staff in transmission prevention measures, and safeguarding themselves by following appropriately the 5 steps of hand hygiene, donning, and doffing of personal protective equipment (PPE). All staff exposed to confirmed COVID-19 cases should report to the infection control officer for exposure risk management. Staff with respiratory symptoms should be clearly instructed to report immediately to the designated physician.

\section{Levels of Personal Protective Equipment}

The following text mentions the low and high COVID risk areas and the appropriate components of PPE essential in each of the risk zones for varying patient related activities as per our institutional guidelines.

High COVID risk areas-Areas where patients are admitted directly from the community to the designated COVID building.

Low COVID risk areas-Areas where the patients are at low risk of COVID based on history and a negative COVID polymerase chain reaction (PCR) result. Although the COVID PCR is negative, PPE based on the type of exposure is required.

The PPEs required have been divided into three levels: 1,2 , and 3 , according to the risk of exposure; level 3 is the highest risk.

\section{Preoperative Assessment}

PPE is recommended for all HCWs in the preanesthetic assessment area. Patients' body temperature should be measured by electronic thermometer. If temperature is higher than $37.3^{\circ} \mathrm{C}$ or if there a significant travel or contact history even if afebrile, the subject must be escorted to the concerned physician for further management. ${ }^{4}$ Strict hand hygiene measures and disinfection of surfaces must be performed between patients.

There is enough evidence in the literature to indicate that a substantial portion of the infected patients are asymptomatic.,7 Hence, it is reasonable to suspect that all patients could be possible carriers of the virus. Currently antibody testing does not have a role in preoperative screening since antibodies develop in the second week of symptoms and not all patients who are infected with severe acute respiratory syndrome coronavirus 2 (SARS-CoV-2) develop detectable antibodies. Additionally, antibody tests can have cross-reaction with other coronaviruses, resulting in false positive results. Additionally, a negative test should be interpreted with caution since sensitivity of SARS-CoV-2 testing is approximately 70 to $90 \% .^{8}$ Considering the high mortality reported in patients undergoing elective procedures during the incubation period, ${ }^{9}$ it is prudent to make all patients undergo PCR test for the COVID-19 virus prior to nonemergent surgery. Testing should be done immediately prior to the surgery, preferably within a 24 hours window to lessen the risk of patient becoming positive during the waiting period. ${ }^{5}$ If a patient tests positive for COVID-19, elective procedures should be deferred until the patient becomes noninfectious as per clinical and laboratory-based criteria that include resolution of respiratory symptoms and fever for at least 72 hours without medication intake and at least 7 days have lapsed since initial symptom presentation even if the symptoms have resolved earlier and two negative tests for COVID-19 done 24 hours apart. ${ }^{8}$

While obtaining the anesthetic consent from the patient, it is important to explain the additional risks of exacerbation of a possible COVID illness, progression to acute respiratory distress syndrome, need for prolonged mechanical ventilation and mechanical circulatory support like intra-aortic balloon pump, extracorporeal membrane oxygenation. ${ }^{9}$

\section{Preoperative Preparation}

Confirmed or suspected 2019-nCoV infected cases should not be brought to holding area. A designated O.R. should be allocated for such cases and signs posted on the doors to minimize staff exposure. Staff surveillance and temperature monitoring to be done twice a day and operating room (OR) staff including the doctors should be divided into batches to ensure availability of adequate HCWs in the event of one batch getting exposed to an undiagnosed COVID patient.

\section{Intraoperative Management}

The following steps are specific to COVID-19 pandemic situation (-Fig. 1). 


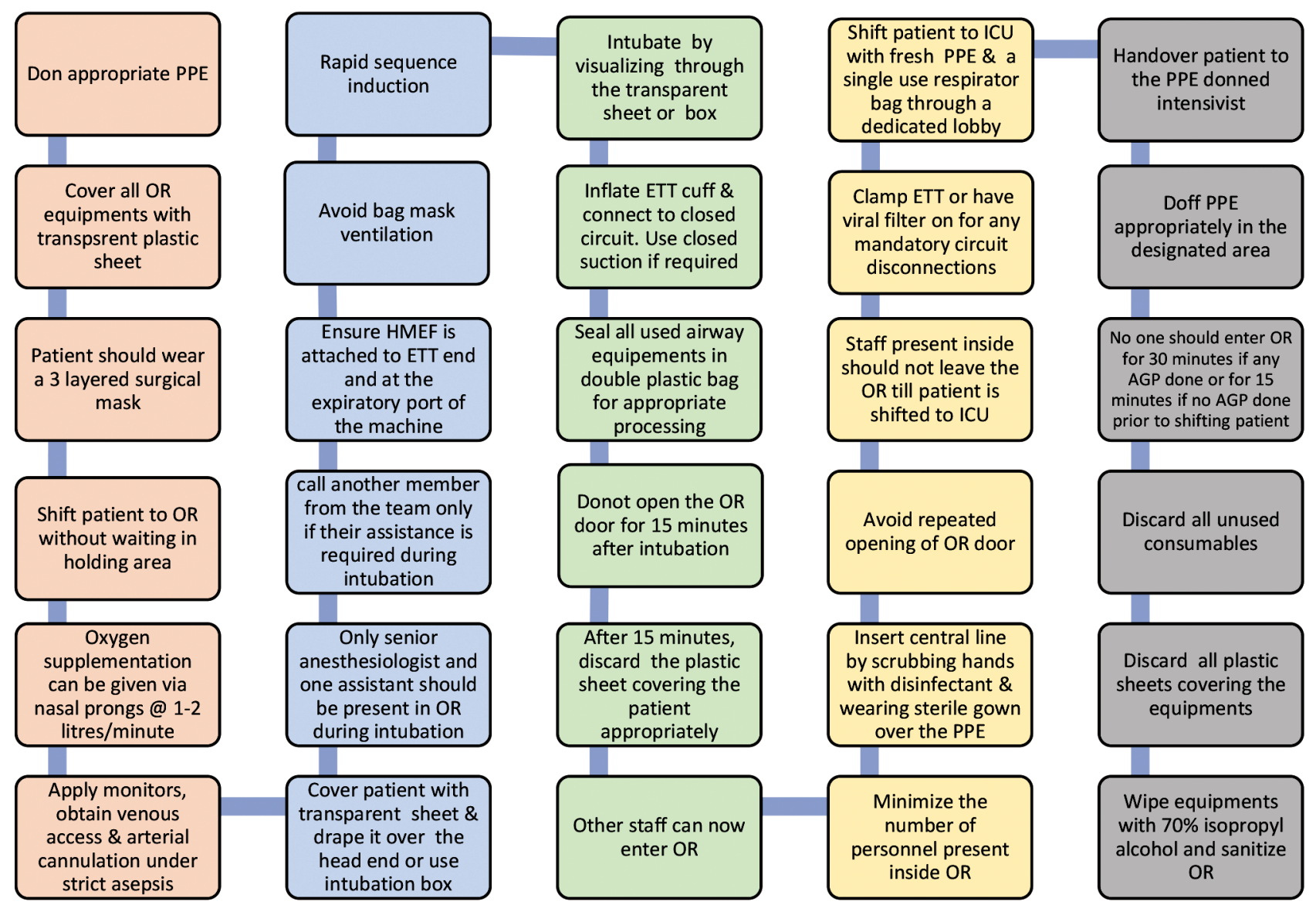

Fig. 1 Operating room (OR) guidelines for coronavirus disease 2019 (COVID-19). ETT, endotracheal tube; ICU, intensive care unit; PPE, personal protective equipment.

- All concerned staff should don PPE, the level of which depends on the elective or emergent nature of the procedure and the patient's COVID-19 status.

- Anesthesia workstation, defibrillator, and other equipment should be covered completely with transparent plastic sheet.

- Routine protocol for cardiac surgery, anesthesia, and cardiopulmonary bypass and World Health Organization (WHO) recommended time-out procedures must be followed diligently.

- Patient should wear a three-layered surgical mask.

- Shift patient to the designated OR without waiting in the holding area to minimize exposure to other patients, staff, and the surroundings.

- Oxygen supplementation, if required, should be given via nasal prongs at 1.5 to $2 \mathrm{~L} / \mathrm{min}$ under the three-layered surgical mask.

- Apply standard monitors, obtain peripheral venous access, and insert arterial line under strict aseptic precautions.

- All the team members should wear double gloves at all times. (if the outer one is soiled, it should be removed and a clean pair should be worn over the inner one)
- Patient should be covered with transparent plastic sheet that is tucked in at the sides and draped over a screen placed high enough at the head end. Alternatively, the transparent intubation box as shown in - Fig. 3 can be used if difficulty in intubation is not anticipated.

- Only the senior anesthesiologist in the team and one assistant should be present in OR to perform intubation (another member from the team to be called in only if their assistance is required during intubation).

- Surgeons and other staff should not be present in the OR at the time of endotracheal intubation.

- Ensure heat and moisture exchanger with filter (HMEF) is attached to the machine end of the endotracheal tube (ETT) and at the expiratory port of the machine since it can filter up to $99 \%$ of airborne particles of 0.3 micron or greater in size. ${ }^{10}$

- Bag mask ventilation should be avoided, and mask with circuit should be held over the surgical mask with 100\% oxygen and minimal sufficient flows.

- Rapid sequence induction with succinyl choline or rocuronium should be done depending on patient condition. 
- Intubation should be done preferably with a video laryngoscope from under the transparent sheet or through the transparent box ( - Fig. 2) while visualizing through it to contain droplet spread.

- Seal all used airway equipment in a double plastic bag and keep secure for appropriate processing.

- ETT cuff should be inflated adequately, then connected to closed circuit. Closed suction system should be used, if ETT suctioning is required.

- The Indian Society of Anesthesiologists (ISA) recommends switching "off" of the air conditioning (AC) for 20 minutes after any AGP, ${ }^{10}$ but our team prefers that AC be kept "on" with laminar flow and high efficiency particulate air (HEPA) filter with 15 to 25 air changes per hour and provision of a

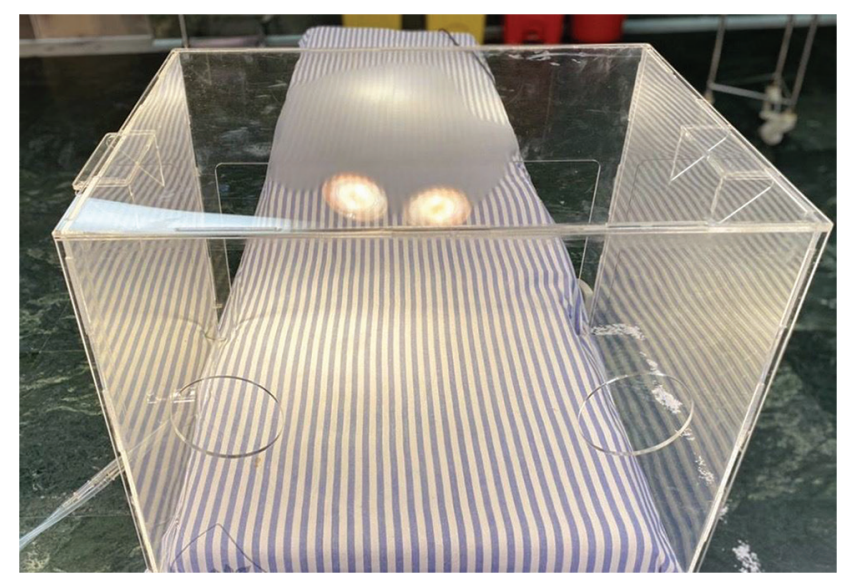

Fig. 2 Intubation box. time gap of 18 to 20 minutes after AGP for satisfactory containment of aerosol spread. It is noteworthy that a similar recommendation is made by www.gov.uk document.

- OR door should not be opened for 15 to 20 minutes after intubation to enable the HEPA filters to remove the aerosol particles since the OR air change rate is around 25 times per hour. ${ }^{11}$

- Plastic sheet over the patient should be removed after 15 minutes of intubation while ensuring that the inner surface of the sheet does not get exposed to any personnel or equipment and discarded appropriately.

- The other staff can enter theater 15 minutes after intubation, but the number of personnel present inside theater must be the bare minimum.

- Avoid repeated opening of OR door to keep the OR pressure and air exchanges regulated.

- Central line insertion should be done by scrubbing hands with disinfectant and wearing sterile surgical gown over the PPE.

- Staff present inside should not leave the OR till patient is shifted to the intensive care unit (ICU).

- Ryle's tube if placed should not be confirmed by auscultation, instead should be confirmed by CXR in the ICU prior to its use.

- Clamp ETT or have the viral filter on for any mandatory breathing circuit disconnections and place the ventilator on standby and turn off gas flows during that period.

- During the conduct of cardiopulmonary bypass, the exhaust gases from the oxygenator should be directed via a tubing to the OR scavenging system.

LEVEL 1 PPE -cap,mask, visor, gown, single pair of gloves

AREAS - outpatient \& inpatient areas (except those listed in level 2), lab (except those handling respiratory samples), blood bank, non aerosol generating procedures

LEVEL 2 PPE -cap, N95 mask, visor, gown, 2 pairs of gloves, shoe cover AREAS - operating rooms(OR), intensive care unit (ICU) during AGP, procedures under general anestrhesia in cardiac catheterization laboratory, endoscopy, bronchoscopy, labor room, dental procedures, AGP like tracheal suctioning in inpatient areas

LEVEL 3 PPE - shoe cover, cap, coverall bodysuit with hood, N95 mask, surgical gown, 2 pairs of gloves, visor

AREAS - COVID building, emergency procedures with unknown or positive COVID status - OR, cardiac catheterization lab, labor ward, endoscopy, bronchoscopy, computed tomography(CT) scan, magnetic resonance imaging(MRI), ambulance, laboratory handling respiratory samples

Fig. 3 Levels and components of personal protective equipment (PPE). 
- After the conduct of the case, the patient is shifted to the ICU cot, and fully covered with a disposable sheet and sides tucked in and monitor can be placed on this sheet. This sheet should be put on by the circulating nurse with fresh gloves and donning fresh PPE (Level 2). One member of the team should put on fresh gloves before handling the single use resuscitator bag. Patient is now wheeled out of the OR, and the manual ventilation is taken over by another member standing in the OR corridor wearing fresh level 2 PPE. This person should have exited the OR a few minutes before transfer, and should have already doffed the PPE used during the case and worn fresh PPE. This second member should now shift and hand over the patient to the PPE donned intensivist. These steps are essential to prevent cross contamination during transfer since most hospitals do not have designated corridors and elevators to shift suspected or positive COVID-19 patients. The PPE worn by the anesthetic team during intubation and airway handling is deemed to be contaminated and should not be worn during transfer to the ICU.

- ETT should have the HMEF attached to it and the breathing circuit is either disconnected distal to the HMEF or the ETT is temporarily clamped and then connected to the single use respirator bag for transfer to the ICU. Hence, the risk of viral aerosolization is negligible during the change of breathing system irrespective of the mode of ventilation during transfer. ISA recommends against the use of transport ventilator to shift patients to the ICU. ${ }^{10}$

- Doffing should be done in the designated place after shifting patient.

- No personnel should enter OR for 30 minutes (if AGP like suctioning or extubation was done immediately prior to shifting patient out of OR) or for 15 minutes. (if no AGP was performed)

- All the unused consumables brought into OR must be discarded.

- All surfaces should be wiped with 70\% isopropyl alcohol and OR should be sanitized. ${ }^{10}$

\section{Pre-extubation Planning}

It is important to keep in mind that the strategies for supporting respiration after extubation like noninvasive ventilation and high flow nasal oxygen are relatively contraindicated. All nonessential staff should exit the room prior to extubation. Prophylactic antiemetics and adequate analgesia should be administered and antitussive agents such as dexmedetomidine, remifentanil, fentanyl, and lignocaine may be considered to prevent aerosol generation due to coughing. Oropharyngeal suctioning should be done with vigilance to contain droplet spread. ${ }^{11}$

\section{Extubation: Steps ${ }^{11}$}

- Extubation should ideally be done in airborne infection isolation rooms with appropriate PPE since it is an AGP. Since such facility is not available in most centers, we recommend the use of transparent box as in $\mathbf{- F i g . ~} \mathbf{2}$ or a transparent plastic sheet to cover the head end during extubation. It is important to limit the number of staff present during the procedure.

- Patient should be kept in 30 degrees headup position.

- Anesthesiologist and assistant should be present behind the head end of the patient.

- Ensure correct face mask size and seal.

- Attach a second airway filter to the facemask.

- Position the ETT to one side of the mouth.

- Position the face mask with second airway filter, using a two-handed technique to ensure a seal over the mouth and nose with the TT exiting under the face mask.

- Endotracheal suctioning when required should be done using closed inline suction catheters.

- Do not administer any positive airway pressure.

- Attempt to extubate at end-expiration while maintaining face mask seal.

- Discard ETT appropriately and provide supplemental oxygen via nasal prongs with minimal essential flows and apply a three-layered surgical mask over it.

\section{Postoperative Care}

- COVID-19, suspected or confirmed, cases should preferably be admitted in negative pressure isolation rooms. If not available, patients should be admitted in single patient rooms in the ICU. ${ }^{3}$

- PPE should be worn at all times, the level of which depends on the patients' COVID status and the type of procedure performed ( - Fig. 3 ).

- Transporting patients outside the ICU should be avoided unless essential.

- Only staff who are directly involved in patient care should be allowed entry into the ICU.

- Protocols should be available in the bedside for hemodynamic support, ventilator care, and supportive management to ensure high-quality patient care with the available staff.

- Strict droplet and contact isolation precautions are mandatory apart from the standard infection control practices for all patients.

- Noninvasive ventilation preferably with a helmet interface and high flow nasal oxygen should be used judiciously and only if absolutely required in recovering cases. ${ }^{3}$ Inhaled medications such as bronchodilators if required should be administered by metered dose inhalers and spacers instead of nebulization to decrease the risk of viral

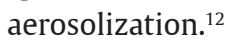

Strict hand hygiene measures, droplet precautions, and use of appropriate PPE must be recommended in the postoperative period if respiratory therapy is clinically indicated. Any AGP should be performed with utmost precautions with strict adherence to the protocol. Providing education on airway clearance techniques, use of incentive spirometry, etc., over the telephone/video calling should be encouraged. 
- Enquiry regarding dietary requirements should be done over the telephone with the concerned staff and use of disposable trays and cutlery should be ensured.

- Use digital media (video conferencing) to communicate with the patient's family and discourage direct visits. ${ }^{3}$

- If there is a high clinical suspicion of COVID-19 in view of the exposure history, clinical symptoms, imaging findings, or the high community prevalence, repeat testing is indicated during the postoperative course. ${ }^{5}$

- However, another recommendation is to repeat PCR testing weekly and to do an exit PCR test on discharge in places with high prevalence of the infection. ${ }^{13}$

\section{Transesophageal Echocardiography}

Transesophageal echocardiography (TEE) use carries heightened risk of viral aerosolization; hence, the following recommendations are given to reduce risk of transmission and cross-contamination. TEE use is indicated only if it is deemed necessary for the surgical procedure (benefits outweigh risks) such as mechanical complications of myocardial infarction, Stanford type A aortic dissection, valvular and perivalvular involvement in infective endocarditis, initiation of mechanical circulatory support, assessment of prosthetic valve, if the patient develops unexplained hemodynamic instability intraoperatively or if a procedural complication is suspected $^{14}$ ( - Fig. 4 ).
-Fig. 5 lists the various steps suggested by American Society of Echocardiography in performing TEE during the COVID-19 outbreak.

\section{Imaging Protocol}

TEE probe insertion, examination, and probe removal should be done by the most experienced echocardiographer donned in full PPE. Pathology oriented abbreviated imaging should be done but it should be extensive enough to detect and evaluate any unexpected but significant pathology. Store the acquired images, report findings in a timely manner, and limit personnel exposure time.

\section{Equipment}

Consider using disposable plastic covers for high touch surfaces (knobs, screen, etc.) to reduce bio burden. Remove all nonessential accessory items from the ultrasound machine. Contamination of surfaces can be avoided by designating one person for probe manipulation and another one for adjusting instrument settings if another PPE donned person is available. Double gloves should be worn at all times, outer gloves should be removed, and inner gloves should be wiped with approved disinfectant solution while undertaking other patient activities. After TEE examination, thoroughly wipe probe, handle, cable, and connector with disinfectant and place in closed container. Wipe outer gloves with disinfectant and then wipe equipment and probe container with disinfectant. Remove

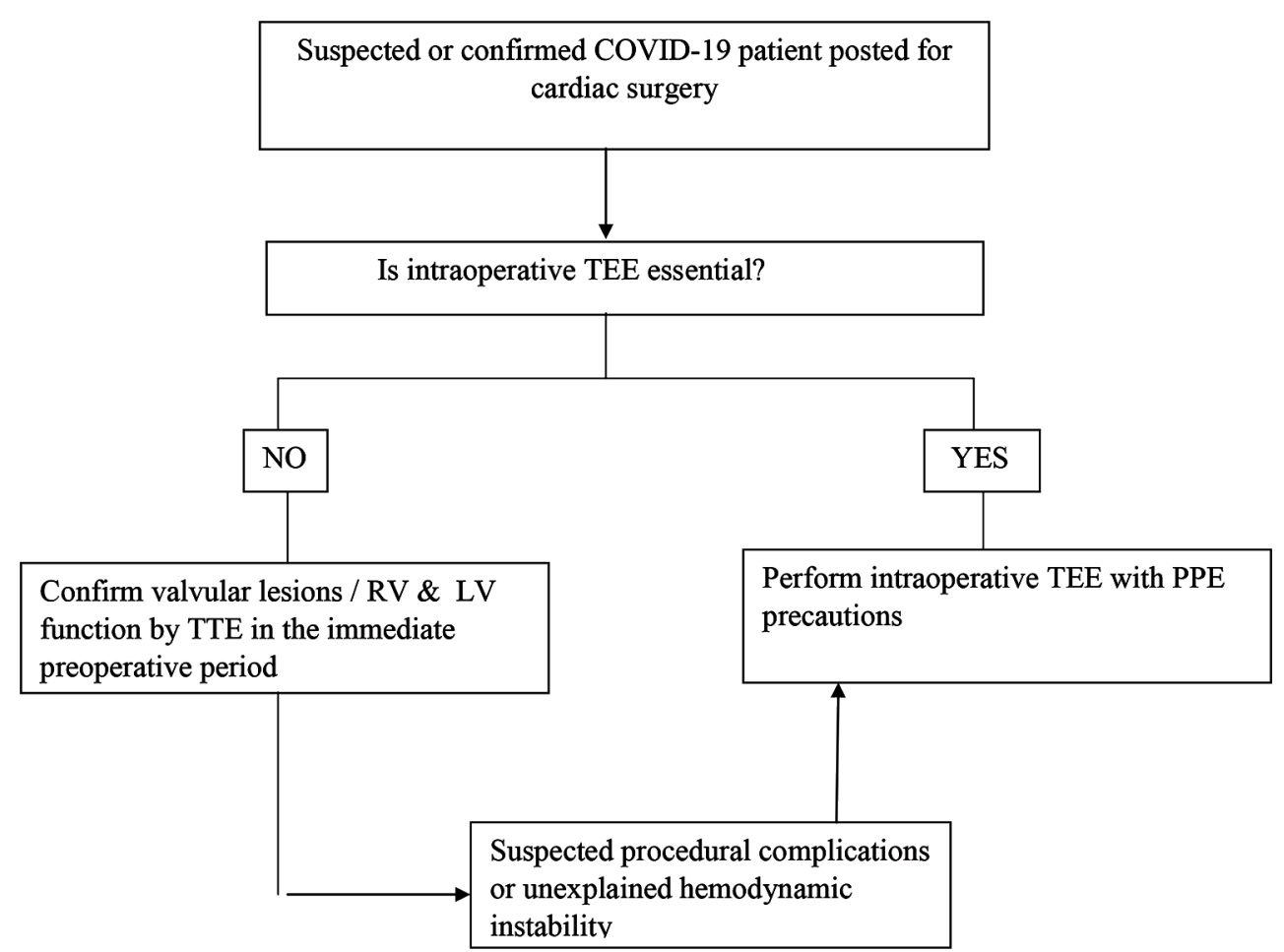

Fig. 4 Indications for intraoperative transesophageal echocardiography (TEE)-American Society of Echocardiography algorithm. COVID-19, coronavirus disease 2019; PPE, personal protective equipment. 


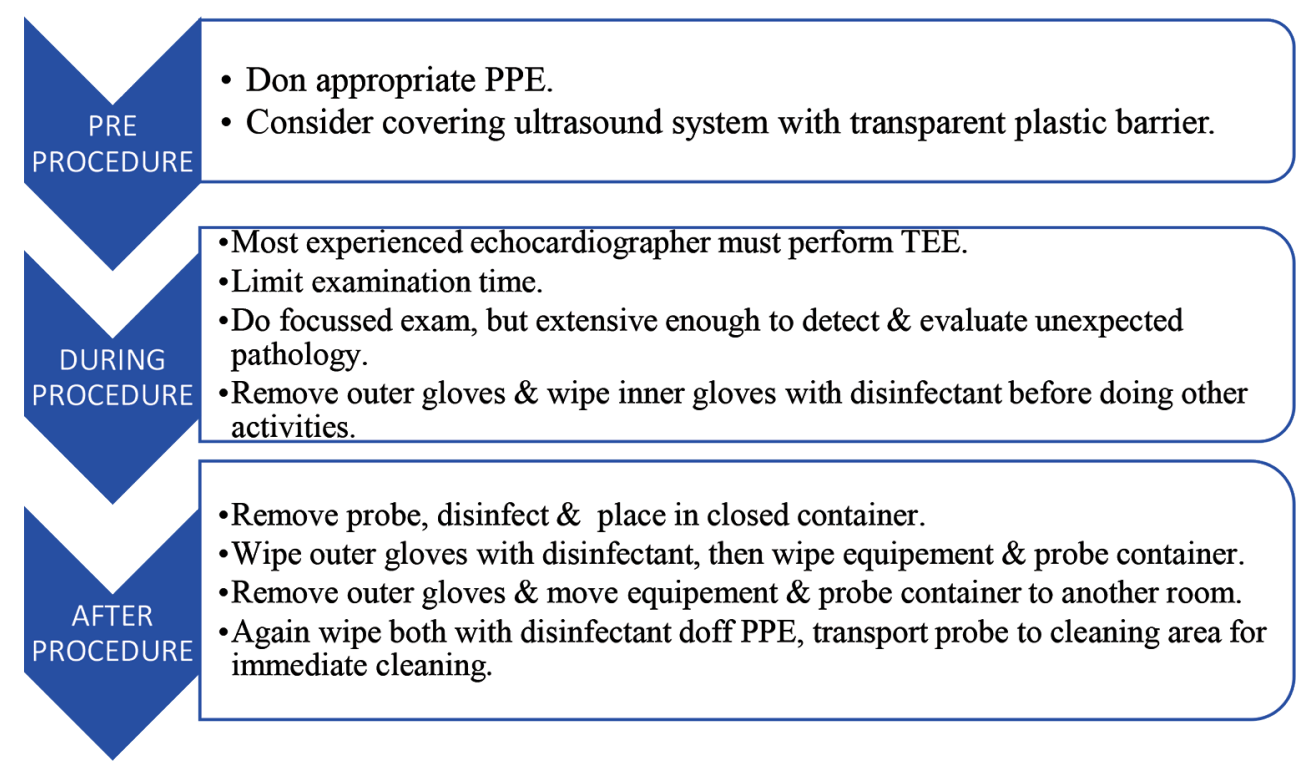

Fig. 5 Stepwise approach to perform transesophageal echocardiography (TEE) as suggested by American Society of Echocardiography. PPE, personal protective equipment.

outer gloves and shift equipment and probe container to another room. Again, wipe equipment surface and probe container with disinfectant, then doff PPE appropriately and transport probe container to the cleaning facility.

\section{Modifications in Cardiopulmonary Resuscitation in COVID-19}

Since CPR involves a high risk of aerosol generation, the following modifications are suggested to the existing algorithm to reduce provider exposure, to adapt ventilation strategies with lower aerosolization risk and to take into account patients' risk factors for survival when assessing resuscitation appropriateness. ${ }^{9}$

- Wear PPE before entering the scene.

- Limit the number of personnel present.

- Consider using mechanical chest compression devices if available.

- Consider non-rebreathing face mask for passive oxygenation for a short duration instead of bag mask device.

- Use HEPA filter when available with tight mask seal for all ventilation.

- Provider with highest chance of first pass success should attempt intubation.

- Pause chest compressions during intubation.

- Consider using videolaryngoscope for intubation.

- Intubate early with cuffed tube to secure and isolate the airway and contain possible aerosol generation.

- Consider supraglottic airway if intubation is delayed.

- Connect to mechanical ventilator when available.

- Avoid disconnection of breathing circuit, but if it is unavoidable, ETT should be clamped prior to disconnection or it should be done distal to the viral filter.

\section{Conclusion}

Extensive measures to reduce person-to-person transmission of COVID-19 are required to control the current outbreak. Special attention and efforts to prevent transmission should be applied to protect healthcare personnel while performing high AGP. Proper institutional guidelines should be followed by all staff during the perioperative period to optimize the quality of care provided to COVID-19 patients and to prevent viral transmission to other patients and HCWs.

\section{Conflict of Interest}

None.

\section{References}

1 World Health Organisation. Available at: www.who.int. Published April 30, 2020. Accessed April 30, 2020

2 bestpractice.bmj.com. Last updated March 30, 2020. Accessed April 29, 2020

3 Mehta Y, Chaudhry D, Abraham OC, et al. Critical care for COVID-19 affected patients: position statement of the Indian Society of Critical Care Medicine. Indian Journal of Critical Care Medicine, https://www.ijccm.org/doi/IJCCM/pdf/10.5005/ jp-journals-10071-23395

4 Chen Y, Liu Y, Gong Y, et al. Perioperative management of patients infected with the novel coronavirus: recommendation from the Joint Task Force of the Chinese Society of Anesthesiology and the Chinese Association of Anesthesiologists Anesthesiology 2020, Vol.132, 1307-1316. doi: https://doi.org/10.1097/ALN.0000000000003301

5 Engelman DT, Lother S, George I, et al. Adult cardiac surgery and the COVID-19 pandemic: aggressive infection mitigation strategies are necessary in the operating room and surgical recovery. Ann Thorac Surg.(2020;doi: https://doi. org/10.1016/j.athoracsur.2020.04.007

6 Gudbjartsson DF, Helgason A, Jonsson H, et al. Spread of SARSCoV-2 in the Icelandic Population. N Engl J Med 2020;382:15 
7 Sutton D, Fuchs K, D’Alton M, Goffman D. Universal screening for SARS-CoV-2 in women admitted for delivery. $\mathrm{N}$ Engl J Med 2020;c2009316

8 American Society of Anaesthesiologists, Anesthesia Patient Safety Foundation Joint Statement on Perioperative Testing for the COVID-19 Virus Published April 28, 2020. Accessed April 30,2020

9 Lei S, Jiang F, Su W, et al. Clinical characteristics and outcomes of patients undergoing surgeries during the incubation period of COVID-19 infection. EClin Med 2020;00:100331

10 Malhotra N, Joshi M, Datta R, Bajwa SJ, Mehdiratta L. Indian Society of Anaesthesiologists (ISA national) advisory \& position statement regarding COVID-19. Indian J Anaesth 2020;64(4):259-263

11 D'Silva DF, McCulloch TJ, Lim JS, et al. Extubation of patients with COVID-19. Br J Anaesth 2020;124(3):314-323

12 Thomas P, Baldwin C, Bissett B, et al. Physiotherapy management for COVID-19 in the acute hospital setting: clinical practice recommendations. J Physiother 2020;2020:S18369553(20)30028-X10.1016/j.jphys.2020.03.011

13 Al-Muharraqi MA. Testing recommendation for COVID-19 (SARS-CoV-2) in patients planned for surgery - continuing the service and 'suppressing' the pandemic. BrJOral Maxillofac Surg 2020;S0266-4356(20)30164-910.1016/j.bjoms.2020.04.014

14 Nicoara A, Maldonado Y, Kort S, et al. Specific considerations for the protection of patients and echocardiography service providers when performing perioperative or periprocedural transesophageal echocardiography during the 2019 novel coronavirus outbreak: council on perioperative echocardiography supplement to the statement of the American Society of Echocardiography. ASEcho.org. Published; 2020

15 Edelson DP, Sasson C, Chan PS, et al. Interim guidance for basic and advanced life support in adults, children and neonates with suspected or confirmed COVID-19: From the Emergency Cardiovascular Care Committee and Get With the Guidelines ${ }^{\circledR}$-Resuscitation Adult and Pediatric Task Forces of the American Heart Association in Collaboration With the American Academy of Pediatrics, American Association for Respiratory Care, American College of Emergency Physicians, The Society of Critical Care Anesthesiologists, and American Society of Anesthesiologists: Supporting Organizations: American Association of Critical Care Nurses and National EMS Physicians Apr 9 doi: 10.1161/CIRCULATIONAHA.120.047463 [Online ahead of print] 